\title{
Regulation of hepatic lipid deposition by phospholipid in large yellow croaker
}

\author{
Zuonan Cai, Kangsen Mai and Qinghui Ai* \\ Key Laboratory of Aquaculture Nutrition and Feed (Ministry of Agriculture) \& Key Laboratory of Mariculture (Ministry of \\ Education), Ocean University of China, 5 Yushan Road, Qingdao, Shandong 266003, People's Republic of China
}

(Submitted 23 March 2017 - Final revision received 27 August 2017 - Accepted 8 September 2017 - First published online 4 December 2017)

\begin{abstract}
Dietary phospholipid (PL) supplementation has been shown to reduce lipid accumulation in the tissues of farmed fish; however, the mechanisms underlying this effect are largely unknown. Thus, the present study was conducted to evaluate the potential impacts of PL on hepatic lipid metabolism both in vivo and in vitro. For in vivo study, four experimental diets - low lipid and low PL diet, as control diet (LL-LP diet, containing $12 \%$ lipid and $1.5 \%$ PL), low-lipid and high-PL diet (containing $12 \%$ lipid and 8\% PL), high-lipid and low-PL diet (HL-LP diet, containing $20 \%$ lipid and $1.5 \%$ PL) and high-lipid and high-PL diet (HL-HP diet, containing $20 \%$ lipid and $8 \%$ PL) - were randomly allocated to four groups of large yellow croaker (Larimichthys crocea) (three cages per group) with similar initial body weight (approximately $8 \mathrm{~g}$ ). For in vitro study, primary hepatocytes isolated from large yellow croaker were incubated either with graded levels of phosphatidylcholine (PC) $(0-250 \mu \mathrm{M})$ or small interfering RNA (siRNA) for CTP: choline phosphate cytidylyltranferase $\alpha$ (CCT $\alpha$ ) (siRNA-CCT $)$. Results showed that survival was independent of dietary treatments $(P>0.05)$. Weight gain and feed efficiency in the HL-HP group were significantly higher than in the LL-LP and HL-LP groups $(P<0.05)$. High level of dietary PL could markedly reduce abnormal hepatic lipid accumulation induced by the HL-LP diet $(P<0.05)$. Similarly, compared with the corresponding controls, a significant decrease/increase in lipid content was observed in primary hepatocytes incubated with PC/siRNA-CCT $(P<0.05)$. High level of dietary PL reversed the HL-LP diet-induced increased levels of mRNA of fatty acid uptake and lipid synthesis related genes $(P<0.05)$. In addition, High level of dietary PL markedly down-regulated the transcript levels of fatty acid oxidation-related genes and enhanced the transcript levels of VLDL assembly-related genes regardless of dietary lipid levels $(P<0 \cdot 05)$. Compared with corresponding controls, primary hepatocytes treated with PC showed significantly higher mRNA expression of lipid synthesis and VLDL assembly-related genes and lower mRNA expression of fatty acid oxidation-related genes, with hepatocytes treated with siRNA-CCT $\alpha$ exhibiting the opposite trend $(P<0.05)$. In summary, these results demonstrated that high level of dietary PL might reverse the HL-LP diet-induced abnormal lipid accumulation in the liver through inhibiting fatty acid uptake and lipid synthesis, together with promoting the lipid export at the transcriptional level. Lipid export-promoting effect of PC was confirmed by in vitro studies. The present study showed for the first time that PL or PC could influence various metabolic pathways to regulate hepatic lipid deposition in fish at least at the transcriptional level.
\end{abstract}

Key words: Large yellow croaker: In vivo and in vitro studies: Phospholipids: Lipid metabolism

Phospholipids (PL) are multifunctional, with phosphatidylcholine (PC) being the most important and abundant class in regular PL sources ${ }^{(1)}$. In mammals, PL are recognised as promising compounds that are useful in the treatment of fatty liver disease ${ }^{(2)}$. Numerous studies have demonstrated that dietary PL can markedly reduce hepatic lipid levels, subsequently alleviating fatty liver disease ${ }^{(3-11)}$. Furthermore, lipid-lowering effects of PL have been suggested to be related with suppression of lipid synthesis and/or enhancement of fatty acid oxidation $^{(6,8,10,11)}$. In addition, a series of in vitro and in vivo studies suggested that when intracellular PC production was inhibited the secretion of VLDL would be attenuated accordingly ${ }^{(12-15)}$. In fish, although it has been reported that supplementation of PL to the diet could prevent lipid accumulation in liver as well ${ }^{(1,16,17)}$, mechanisms related are still elusive.

Large yellow croaker (Larimichthys crocea) is a commercially important fish species in China because of its delicious taste. In the past decade, high-lipid diet has been extensively used in the culture of this fish species owing to its protein sparing effect ${ }^{(18)}$. However, high-lipid diet can induce abnormal lipid accumulation in fish liver, giving rise to increased inflammation and disturbed metabolism ${ }^{(19,20)}$, which are similar to those observed

Abbreviations: $A C O$, acyl-CoA oxidase; $A P O B 100$, apoB100; CCT $\alpha$, CTP: choline phosphate cytidylyltranferase $\alpha$; $C D 36$, cluster of differentiation 36; CPT1: carnitine palmitoyltransferase 1; DGAT2, acyl-CoA: diacylglycerol acyltransferase 2; FABP, fatty acid binding protein; $F A S$, fatty acid synthase; HL-HP, high lipid and high phospholipid; HL-LP, high lipid and low phospholipid; LL-HP, low lipid and high phospholipid; LL-LP, low lipid and low phospholipid; MTP, microsomal TAG transfer protein; PC, phosphatidylcholine; PL, phospholipid; SCD1, stearoyl-CoA desaturase 1; siRNA, small interfering RNA; SREBP1, sterolregulatory element binding protein 1.

* Corresponding author: Q. Ai, fax +8653282031943, email qhai@ouc.edu.cn, aiqinghui@163.com 
containing $10 \%$ FBS, $100 \mathrm{U} / \mathrm{ml}$ penicillin and $100 \mu \mathrm{g} / \mathrm{ml}$ streptomycin). Primary hepatocytes were seeded into six-well culture dishes ( $2 \mathrm{ml} /$ well) (Corning) at a density of $2 \times 10^{6}$ cells/well and incubated at $28^{\circ} \mathrm{C}$ in $5 \% \mathrm{CO}_{2}$.

\section{Treatment of primary hepatocytes}

Isolation and culture of primary hepatocytes were conducted according to the protocols mentioned above. During culture as above, the medium was replaced with new complete medium every $24 \mathrm{~h}$. After $2 \mathrm{~d}$ of culture, primary hepatocytes were serumstarved overnight. For experiment one, primary hepatocytes were incubated with graded concentrations of PC (0 (control), 50, 100, 150,200 and $250 \mu \mathrm{m}$, respectively) for $12 \mathrm{~h}$. For experiment two, hepatocytes were transfected with small interfering RNA (siRNA) duplexes (5'-Chol, 2'-Ome) for CTP: choline phosphate cytidylyltranferase $\alpha(C C T \alpha)$ (siRNA-CCT $\alpha)$ or negative control (NC) (siRNA-NC) (GenePharma), which were named CCT $\alpha$ group and control group, respectively. The sequences of CCT $\alpha$ siRNA duplexes were as follows: sense sequence, 5'-GGG UGU AUG CAG AUG GCA UTT-3'; anti-sense sequence, 5'-AUG CCA UCU GCA UAC ACC CTT-3'. The sequences of NC siRNA duplexes were as follows: sense sequence, 5'-UUC UCC GAA CGU GUC ACG UTT-3'; anti-sense sequence, 5'-ACG UGA CAC GUU CGG AGA ATT-3'. The delivery of siRNA duplexes was carried out using Lipofectamine ${ }^{\circledR}$ RNAiMAX Transfection Reagent (Invitrogen) according to the manufacturer's instructions. Primary hepatocytes were incubated with siRNA-lipid complex for $48 \mathrm{~h}$. The final concentration of siRNA used was $25 \mathrm{pmol} /$ well. For in vitro study, each treatment was replicated four times $(n$ 4). Cells were harvested after treatment for the indicated time.

\section{Biochemical analysis}

Crude protein of the experimental diets was determined according to the Kjeldahl method (Kjeltec 2300; Foss Tecator) and estimated by multiplying $\mathrm{N}$ by $6 \cdot 25$.
Crude lipid contents of the experimental diets and whole fish body were determined by diethyl ether extraction using the Soxhlet method (Soxhlet Extraction System B-811; Buchi). Hepatic lipid contents of fish were measured based on the described procedures ${ }^{(22)}$. TAG contents in the primary hepatocytes were determined by TAG Assay Kit according to the manufacturer's protocols (Polygen).

PL contents of the experimental diets were measured by determining $\mathrm{P}$ content $(\mathrm{P} \times 25)$ using molybdenum blue $\operatorname{method}^{(23)}$. In brief, about $100 \mathrm{mg}$ of freeze-dried samples were digested with nitric acid and perchloric acid and added with molybdenum blue reagent. The $\mathrm{P}$ content can be calculated by assaying the absorbance at $830 \mathrm{~nm}$ with the UV-2401PC spectrophotometer (Shimadzu Corporation). PC content in primary hepatocytes was measured using the PC Assay Kit (Sigma) according to the manufacturer's instructions.

\section{Quantitative real-time PCR}

Complementary DNA preparation and quantitative real-time PCR were conducted on the procedures described by Zuo et $a l .{ }^{(24)}$. Primers for each target gene were directly synthesised based on the corresponding sequences in published papers $^{(20,25)}$ (Table 2). The relative expression ratio was determined by the formula $2^{-\Delta \Delta C_{t}(26)}$. 18s rRNA, $\beta$-actin, glyceraldehyde-3-phosphate dehydrogenase (GAPDH), elongation factor $1 \alpha$ and ubiquitin were ranked according to their stability using geNorm (version 3.5) and NormFinder algorithms $^{(27,28)}$ and $\beta$-actin was used as the reference gene. For in vivo study, the relative mRNA expression of target genes in fish fed the LL-LP diet was selected as the calibrator; for in vitro study, the relative mRNA expression of target genes in primary hepatocytes incubated with $0 \mu \mathrm{M}$ PC was selected as the calibrator.

Table 2. Primer pair sequences for quantitative real-time-PCR

\begin{tabular}{lll}
\hline Target genes & Forward (5'-3') & Reverse (5'-3') \\
\hline LPL & GAGAGGATTCATCTGCTGGGTTAC & ACATCAACAAACTGGGCGTCATC \\
HL & GCCGTCCATCTATTCATTGACTCTC & GCCACTGGAACCTTCTTGATATTG \\
CD36 & GAGCATGATGGAAAATGGTTCAAAG & CTCCAGAACTCCCTTTCACCTTAG \\
FATP1 & CAACCAGCAGGACCCATTACG & CATCCATCACCAGCACATCACC \\
FABP3 & CCAAACCCACCACTATCATCTCAG & GCACCATCTTTCCTCCTCTATTG \\
FABP10 & CAATGGAACATGGCAGGTTACG & TGATTGGCTTGATGTCCTTGGC \\
FABP11 & CAGGTGGGCAATCGGACCAA & GGCTCGTTGAGCTTGAACTTGA \\
FAS & CAGCCACAGTGAGGTCATCC & TGAGGACATTGAGCCAGACAC \\
SCD1 & AAAGGACGCAAGCTGGAACT & CTGGGACGAAGTACGACACC \\
DGAT2 & TTCGGTGCTTTCTGCAACTTCG & AAGGATGGGGAAGCGGAAGT \\
SREBP1 & TCTCCTTGCAGTCTGAGCCAAC & TCAGCCCTTGGATATGAGCCT \\
CPT1 & GCTGAGCCTGGTGAAGATGTTC & TCCATTTGGTTGAATTGTTACTGTCC \\
ACO & AGTGCCCAGATGATCTTGAAGC & CTGCCAGAGGTAACCATTTCCT \\
$P P A R a$ & GTCAAGCAGATCCACGAAGCC & TGGTCTTTCCAGTGAGTATGAGCC \\
$M T P$ & ATGTCCAAAATGTTCTCCATGTCTG & ATGTCAATAGCCAACCCTCCTTG \\
APOB100 & AGAGTGTTGTCCAGGATAAAGATGC & CAGGGCTCAGGGTCTCAGTC \\
CCTa & CGCCAGAGTTTCTCGCAAGACATCG & CGTGGACAAGGTGAAGAGGAAGGTGC \\
$\beta-A C T I N$ & CTACGAGGGTTATGCCCTGCC & TGAAGGAGTAACCGCGCTCTGT \\
\hline
\end{tabular}

$L P L$, lipoprotein lipase; $H L$, hepatic lipase; $C D 36$, cluster of differentiation 36; FATP1, fatty acid transport protein 1; FABP, fatty acid binding protein; $F A S$, fatty acid synthase; $S C D 1$, stearoyl-CoA desaturase 1; DGAT2, acyl-CoA: diacylglycerol acyltransferase 2; SREBP1, sterol-regulatory element binding protein 1; CPT1: carnitine palmitoyltransferase 1; ACO, acyl-CoA oxidase; MTP microsomal TAG transfer protein; APOB100, apo B100. 


\section{Western blotting}

Total protein was extracted using Tissue or Cell Total Protein Extraction Kit (Sangon Biotech). Quantification of the concentrations was conducted by a Noninterference Protein Assay Kit (Sangon Biotech). Protein was separated by denaturing SDS-PAGE, and then transferred to a polyvinylidene difluoride membrane. Next, the membranes were incubated with CCT $\alpha$ or GAPDH antibody (\#4454 and \#2118; Cell Signaling Technology) before horseradish peroxidase-conjugated secondary antibodies (A0208; Beyotime Institute of Biotechnology) were added. Protein bands were visualised using ECL reagents (Beyotime Institute of Biotechnology).

\section{Statistical analysis}

Software SPSS 17.0 (SPSS Incorporation) was used for all statistical evaluations. The results are expressed as mean values with their standard errors. Data were analysed using two-tailed Student's $t$ test or one-way ANOVA, followed by Tukey's test. Polynomial contrasts (linear, quadratic and cubic) were used to test the effects of PC concentrations on the various variables measured in the primary hepatocytes. The level of significance was set at $P<0 \cdot 05$. Densitometry after western blotting was quantified by NIH Image 1.63 software (National Institutes of Health) and then normalised by GAPDH.

\section{Results}

Results for in vivo study

Survival and growth parameters for large yellow croaker fed the experimental diets. There were no significant differences in survival among dietary treatments $(P>0 \cdot 05)$. Fish in the HL-HP group had significantly higher weight gain and feed efficiency (FE) than in the LL-LP and HL-LP groups $(P<0 \cdot 05)$, whereas there were no significant differences between the LL-LP and HL-LP groups $(P>0.05)$. Feed intake (FI) in the HL-HP group was comparable to that in the HL-LP group $(P>0.05)$, but was significantly lower than that in the LL-LP group $(P<0.05)$. In addition, there were no significant differences in weight gain, FI and FE between the LL-LP and LL-HP groups (Table 3).

Lipid contents of the whole body and liver in large yellow croaker fed the experimental diets. No significant differences in the whole-body lipid contents were observed among the LL-LP, HL-LP and HL-HP groups ( $P>0.05)$. Compared with the LL-LP group, lipid levels in the livers were significantly higher in the HL-LP group $(P<0.05)$, which was indicative of abnormal hepatic lipid accumulation. High dietary PL diminished the HL-LP diet-mediated up-regulation of hepatic lipid level $(P<0 \cdot 05)$. Although no significant differences were detected, the LL-HP diet tended to down-regulate lipid content of the liver compared with the LL-LP group $(P>0 \cdot 05)$ (Table 4$)$.

Expression of genes related to fatty acid uptake in the liver of large yellow croaker fed the experimental diets. Compared with the LL-LP diet, the HL-LP diet significantly increased the mRNA expression levels of lipoprotein lipase (LPL), hepatic lipase (HL), cluster of differentiation 36 (CD36), fatty acid transport protein 1 (FATP1) and fatty acid binding protein 11 (FABP11) $(P<0.05)$, which were reversed by the dietary

Table 3. Survival and growth parameters for large yellow croaker fed the experimental diets

(Mean values with their standard errors; $n 3$ )

\begin{tabular}{|c|c|c|c|c|c|c|c|c|}
\hline & \multicolumn{2}{|c|}{ LL-LP } & \multicolumn{2}{|c|}{ LL-HP } & \multicolumn{2}{|c|}{ HL-LP } & \multicolumn{2}{|c|}{ HL-HP } \\
\hline & Mean & SEM & Mean & SEM & Mean & SEM & Mean & SEM \\
\hline Survival (\%)‡ & $91 \cdot 11$ & 0.56 & $91 \cdot 11$ & $1 \cdot 11$ & 89.44 & 0.56 & $90 \cdot 56$ & $1 \cdot 11$ \\
\hline Initial weight (g) & 8.39 & 0.04 & 8.37 & 0.05 & 8.37 & 0.05 & 8.41 & 0.02 \\
\hline Final weight $(\mathrm{g})$ & $46 \cdot 75$ & 1.82 & 47.96 & 0.80 & $48 \cdot 27$ & 1.98 & $56 \cdot 20^{*} \dagger$ & 1.52 \\
\hline Weight gain $(\mathrm{g}) \S$ & $38 \cdot 37$ & 1.86 & 39.59 & 0.79 & 39.91 & 1.96 & $47 \cdot 80^{*} \dagger$ & 1.51 \\
\hline Feed intake $\|$ & 1.44 & 0.04 & 1.48 & 0.04 & 1.39 & 0.03 & $1 \cdot 31^{*}$ & 0.03 \\
\hline Feed efficiency(\%)ף & $75 \cdot 31$ & $3 \cdot 12$ & 74.25 & 3.54 & 77.48 & $2 \cdot 12$ & $87 \cdot 66^{\star} \dagger$ & 1.48 \\
\hline
\end{tabular}

LL-LP, low lipid and low phospholipid; LL-HP, low lipid and high phospholipid; HL-LP, high lipid and low phospholipid; HL-HP, high lipid and phospholipid.

*Significant differences between the LL-LP and the other treated groups $(P<0.05$; two-tailed Student's $t$ test).

† Significant difference between the HL-LP and HL-HP groups $(P<0.05$; two-tailed Student's $t$ test).

$\ddagger$ Survival $(\%)=100 \times$ final fish number/initial fish number.

$\S$ Weight gain $=$ final weight - initial weight.

\| Feed intake $(/$ day $)=$ feed consumption $(\mathrm{g}) /(\mathrm{d} \times($ final body weight + initial body weight $) / 2)$.

II Feed efficiency $=$ wet weight gain $(\mathrm{g}) /$ feed consumption $(\mathrm{g})$.

Table 4. Lipid contents of the whole body and liver for large yellow croaker fed the experimental diets (\%, wet weight)

(Mean values with their standard errors; $n$ 3)

\begin{tabular}{|c|c|c|c|c|c|c|c|c|}
\hline & \multicolumn{2}{|c|}{ LL-LP } & \multicolumn{2}{|c|}{ LL-HP } & \multicolumn{2}{|c|}{ HL-LP } & \multicolumn{2}{|c|}{ HL-HP } \\
\hline & Mean & SEM & Mean & SEM & Mean & SEM & Mean & SEM \\
\hline Whole body & $9 \cdot 88$ & 0.35 & $8.42^{*}$ & 0.28 & $10 \cdot 21$ & 0.48 & $9 \cdot 34$ & 0.29 \\
\hline Liver & $25 \cdot 61$ & 1.36 & 23.63 & 1.63 & $37.41^{*}$ & 4.14 & $28.95 \dagger$ & 1.51 \\
\hline
\end{tabular}

LL-LP, low lipid and low phospholipid; LL-HP, low lipid and high phospholipid; HL-LP, high lipid and low phospholipid; HL-HP, high lipid and phospholipid.

*Significant differences between the LL-LP and the other treated groups $(P<0.05$; two-tailed Student's $t$ test).

† Significant difference between the HL-LP and HL-HP groups $(P<0.05$; two-tailed Student's $t$ test). 
(a)

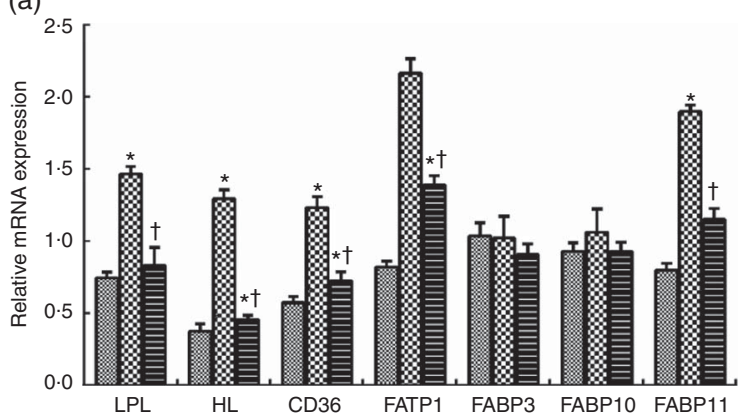

(c)

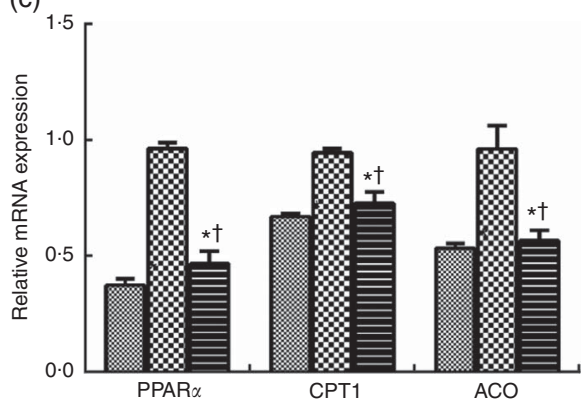

(b)

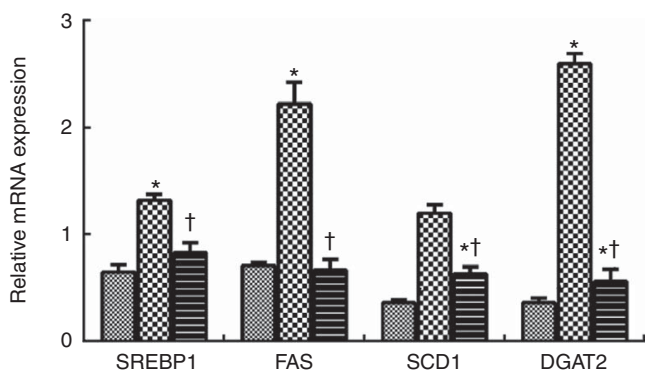

(d)

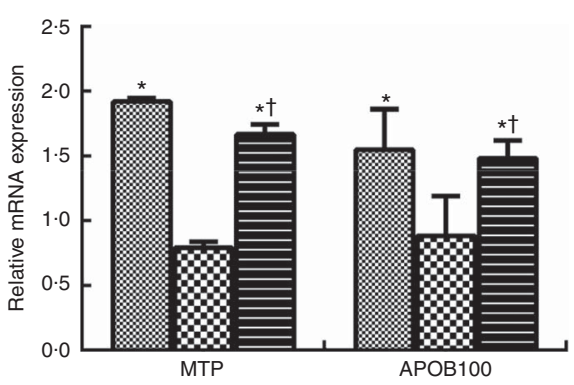

Fig. 1. Expression of genes related to fatty acid uptake (a), lipid synthesis (b), fatty acid oxidation (c) and VLDL assembly (d) in liver of large yellow croaker. Values are means $(n 3)$, with their standard errors represented by vertical bars. The relative mRNA expression of target genes in the low lipid and low phospholipid (LL-LP) group was selected as the calibrator. 图, low lipid and high phospholipid (LL-HP); 圈, high lipid and low phospholipid (HL-LP); 目, high lipid and phospholipid (HL-HP); LPL, lipoprotein lipase; $H L$, hepatic lipase; $C D 36$, cluster of differentiation 36; FATP1, fatty acid transport protein 1; FABP, fatty acid binding protein; CPT1: carnitine palmitoyltransferase 1; ACO, acyl-CoA oxidase; APOB100, apo B100. Significance was evaluated by two-tailed Student's $t$ test. Significant differences between the LL-LP and the other treated groups: ${ }^{*} P<0.05$; significant difference between the HL-LP and HL-HP groups: $\dagger P<0.05$.

inclusion of high PL $(P<0 \cdot 05)$. No significant differences were detected in FABP3 and FABP1O mRNA expression between the LL-LP and HL-LP groups, and similar results were observed between the HL-LP and HL-HP groups $(P>0.05)$. Among all the seven proteins involved in fatty acid uptake in the liver, only $H L$ and $C D 36$ mRNA levels displayed a significant decrease in the LL-HP group than in the LL-LP group $(P<0.05)$ (Fig. 1(a)).

Expression of genes related to lipid synthesis in the liver of large yellow croaker fed the experimental diets. Compared with the LL-LP group, the transcript levels of sterol-regulatory element binding protein 1 (SREBP1), fatty acid synthase (FAS) and acyl-CoA: diacylglycerol acyltransferase 2 (DGAT2) were significantly increased by about $0 \cdot 36$-fold, 1.22 -fold and 1.59 fold in the HL-LP group, respectively $(P<0.05)$, but there were no significant differences in stearoyl-COA desaturase 1 (SCD1) mRNA expression between these two groups $(P>0.05)$. Dietary incorporation of high PL significantly inhibited high lipidinduced up-regulation in the transcript levels of SREBP1, FAS and DGAT2 $(P<0.05)$. In addition, fish in the HL-HP group displayed significantly lower mRNA expression level of $S C D 1$ than in the HL-LP group $(P<0.05)$. The mRNA expression levels of $S C D 1$ and DGAT2 were significantly lower in fish fed the LL-HP diet than those fed the LL-LP diet $(P<0 \cdot 05)$ (Fig. 1(b)).

Expression of genes related to fatty acid oxidation in the liver of large yellow croaker fed the experimental diets. No significant differences were observed in key genes participating in fatty acid oxidation (PPAR $\alpha$ (PPAR $)$, carnitine palmitoyltransferase 1 (CPT1) and acyl-COA oxidase (ACO)) between the LL-LP and HL-LP groups $(P>0.05)$. Fish in the HL-HP group had significantly lower mRNA expression levels of PPAR $\alpha, C P T 1$ and $A C O$ compared with the HL-LP group $(P<0.05)$. The mRNA expression levels of PPAR $\alpha, C P T 1$ and $A C O$ of fish fed the LL-HP diet were significantly lower than those of fish fed the LL-LP diet $(P<0 \cdot 05)$ (Fig. 1(c)).

Expression of genes related to VLDL assembly in the liver of large yellow croaker fed the experimental diets. There were no significant differences in microsomal TAG transfer protein $(M T P)$ and apo B100 (APOB100) mRNA expression levels between the LL-LP and HL-LP groups $(P>0.05)$. Compared with the HL-LP group, the transcript levels of MTP and APOB10O were significantly higher in the HL-HP group $(P<0 \cdot 05)$. The mRNA expression levels of these two proteins were significantly higher in the LL-HP group compared with those in the LL-LP group $(P<0.05)$ (Fig. $1(\mathrm{~d}))$.

\section{Results for in vitro study}

Effects of graded phosphatidylcholine levels on TAG contents and mRNA expression of genes related to lipid metabolism in primary hepatocytes of large yellow croaker. TAG contents decreased with the increasing levels of PC from 0 to $150 \mu \mathrm{M}$; although it then increased a little with the increasing levels of PC from 150 to $250 \mu \mathrm{m}$, TAG contents in the 200 and 


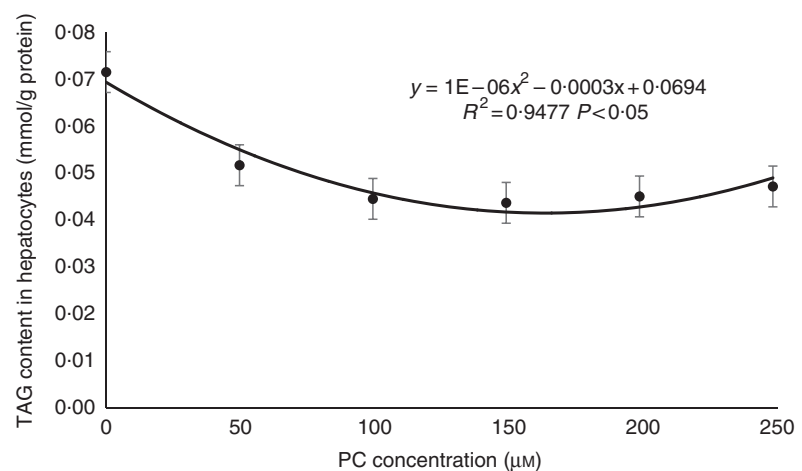

Fig. 2. Regression analyses about TAG contents in primary hepatocytes of large yellow croaker in response to graded concentrations of phosphatidylcholine (PC). Values are means $(n 4)$, with their standard errors.

$250 \mu \mathrm{m}$ PC groups were still higher than in the $0 \mu \mathrm{M}$ PC groups (Fig. 2).

The mRNA expression levels of genes related to lipid synthesis, including SREBP1, FAS, SCD1 and DGAT2, increased with the increasing levels of PC (Fig. 3(a)).

The mRNA expression levels of genes related to fatty acid oxidation, including $P P A R \alpha, C P T 1$ and $A C O$, decreased with the increasing levels of PC (Fig. 3(b))

The mRNA expression levels of genes related to VLDL assembly, including $M T P$ and $A P O B 100$, increased with increasing levels of PC (Fig. 3(c)).

Effects of CTP: choline phosphate cytidylyltranferase $\alpha$ knockdown on CTP: choline phosphate cytidylyltranferase $\alpha$ mRNA expression, CTP: choline phosphate cytidylyltranferase $\alpha$ protein expression, $P C$ and TAG contents and mRNA expression of genes related to lipid metabolism in primary hepatocytes of large yellow croaker. CCT $\alpha$ is the rate-limiting enzyme during de novo biosynthesis of PC in CDP-choline pathway in fish, as well as in mammals ${ }^{(29)}$. Thus, in the present study, CCTa knockdown was applied for inhibition of endogenous PC production in primary hepatocytes. CCT $\alpha$ mRNA and protein levels were significantly reduced by about $50 \%$ in primary hepatocytes transduced with siRNA-CCT $\alpha(P<0.05)$ (Fig. 4(a) and (b)). As a consequence, siRNA-CCT $\alpha$ significantly decreased PC content in primary hepatocytes, confirming the successful knockdown of CCT $\alpha$ in the present study $(P<0.05)$ (Fig. 4(c)).

Primary hepatocytes transfected with siRNA-CCT $\alpha$ displayed significantly higher TAG content than those transfected with siRNA-NC $(P<0.05)$ (Fig. 4(d)).

The mRNA levels of SREBP1, FAS, SCD1 and DGAT2 were reduced by about 0.36-fold, 0.28-fold, 0.39-fold and 0.34-fold, respectively, in primary hepatocytes transfected with siRNA-CCTa compared with those in the control group $(P<0.05)$ (Fig. 5(a)).

The mRNA levels of PPAR,$C P T 1$ and $A C O$ were increased by about 0.41 -fold, 0.45 -fold and 0.30 -fold, respectively, in the CCTagroup compared with those in the control group $(P<0.05)$ (Fig. 5(b)).

The mRNA levels of MTP and $A P O B 100$ were reduced by about 0.60 -fold and 0.34-fold, respectively, in the CCT $\alpha$ group compared with those in the control group $(P<0.05)$ (Fig. 5(c)).

\section{Discussion}

In the present study, no significant differences in survival and weight gain were observed between the LL-LP and LL-HP groups, which was consistent with studies conducted in juvenile large yellow croaker $^{(1)}$, Atlantic salmon ${ }^{(30)}$ and white sturgeon $^{(31)}$. In contrast, PL has been reported to exert beneficial effects on survival and growth performance in various fish species during their larval stage $\mathrm{e}^{(1,30-35)}$. The possible reason for this discrepancy was that PL de novo synthesis was compromised in fish larvae, as many genes related with PL synthesis exhibited lower expression during the earlier developmental stage of fish ${ }^{(36)}$. There were also no significant differences in survival and weight gain between the LL-LP and HL-LP groups, which was supported by the findings of Yan et al. ${ }^{(20)}$ and Wang et al. ${ }^{(37)}$ in the same species. Interestingly, in the present study, weight gain in the HL-HP group was significantly higher than in the LL-LP and HL-LP groups. An increase in FE in the HL-HP group might account for this observation. One explanation for these results was that high dose of PL could optimise lipid metabolism, and then facilitate lipid utilisation in fish fed the HL-LP diet, which in turn resulted in higher $\mathrm{FE}$ and better growth.

The important role of PL in alleviating abnormal lipid deposition has also been established in fish. Hepatic lipid contents markedly decreased from 21 to $16 \%$ as dietary PL supplementation increased from $6 \%$ to $18 \%$ in large yellow croaker $^{(1)}$. The addition of PL to the diet caused a reduction in the number of lipid vacuoles in hepatocytes in catfish ${ }^{(16)}$. In this study, high dietary PL notably inhibited high lipid-induced increased hepatic lipid levels. Besides, 50 to $250 \mu \mathrm{m}$ PC significantly reduced TG contents in primary hepatocytes and suppression in cellular PC production by CCT $\alpha$ knockdown resulted in an increase in TG content in primary hepatocytes. Taken together, these results from both in vivo and in vitro studies observed in the present study not only confirmed the lipid-reducing effects of PL as observed in the in vivo study but also further emphasised a key role of PC in reducing hepatic lipid accumulation. In agreement with these results in fish, in mammals, Liu et $a l^{(10)}$ found that PL significantly decreased hepatic TAG level in mice fed the high-fat diet. Similarly, the findings of Liu et al. ${ }^{(11)}$ and Buang et al. ${ }^{(6)}$ suggested that PC could markedly ameliorate hepatic TG accumulation in rats with nonalcohol fatty liver disease induced by orotic acid. In addition, Jacobs et al. ${ }^{(15)}$ reported an accumulation of intracellular TAG in CCT $\alpha$-deficient hepatocytes isolated from mouse.

However, to our knowledge, the mechanisms underpinning the lipid-reducing effects of PL in fish are not fully understood. As PL has been shown to reduce hepatic lipid contents by decreasing lipid uptake and synthesis and increasing lipid catabolism and export in mammals ${ }^{(6,8,10-15)}$, it was postulated that for fish fed diets with PL or primary hepatocytes incubated with PC, the expression of genes related with fatty acid uptake and lipid synthesis would also be lower, and the expression of genes related with fatty acid oxidation and lipid export would also be higher, which together resulted into lower hepatic lipid contents. Thus, in the present study, the effects of PL on hepatic lipid metabolism, including fatty acid uptake, lipid synthesis, fatty acid oxidation and lipid export, were explored both in vivo and 

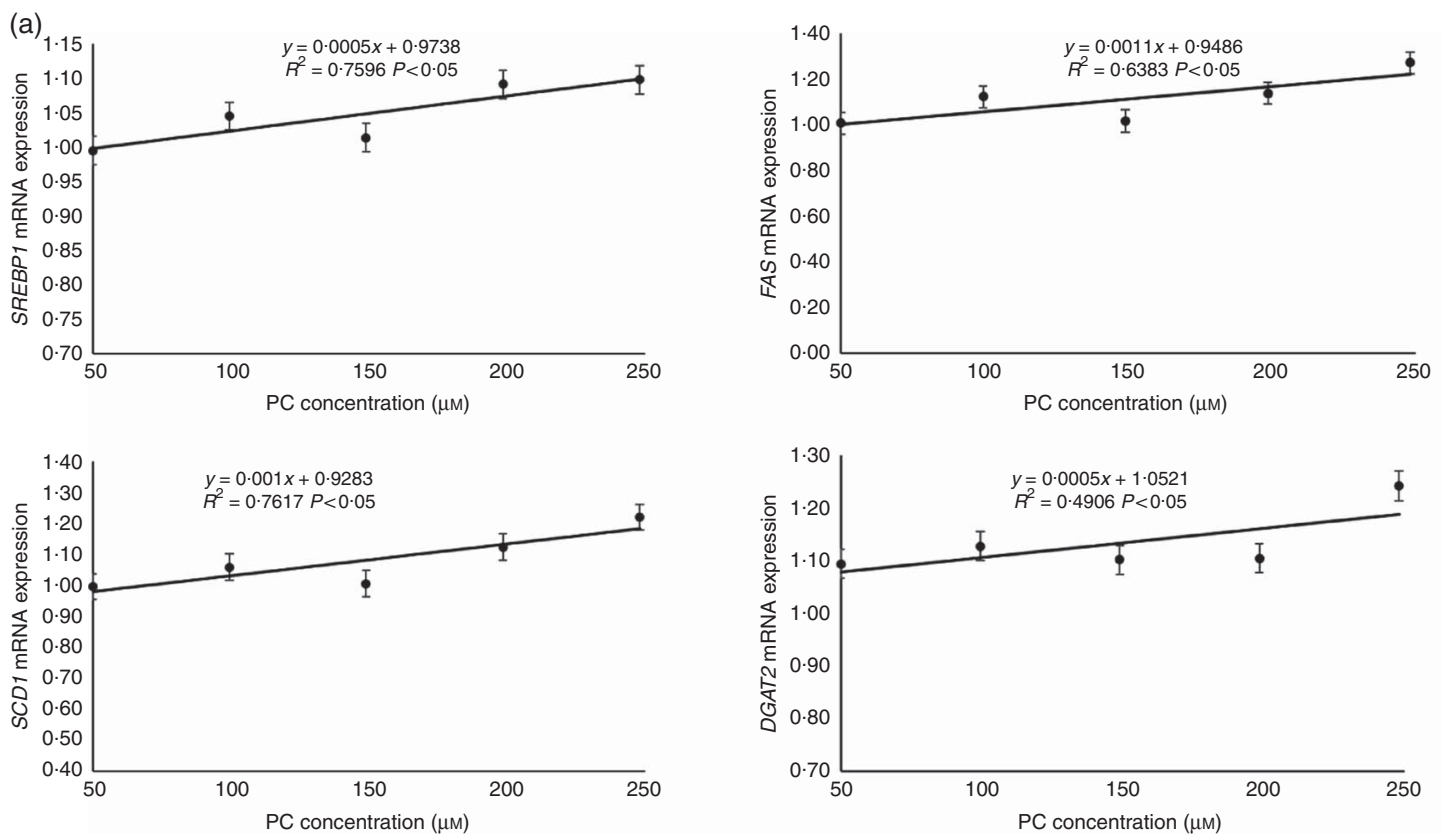

(b)
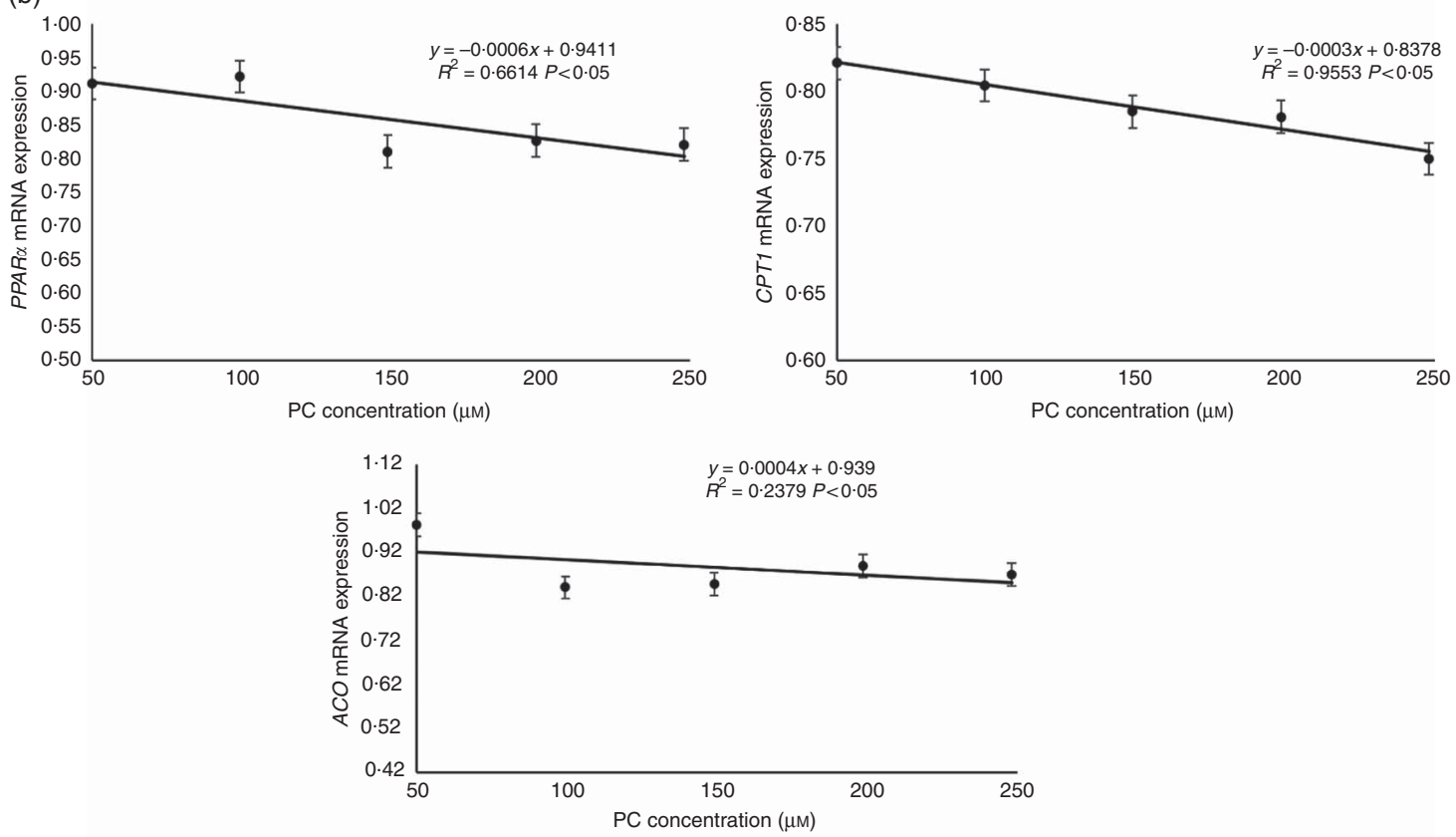

(c)
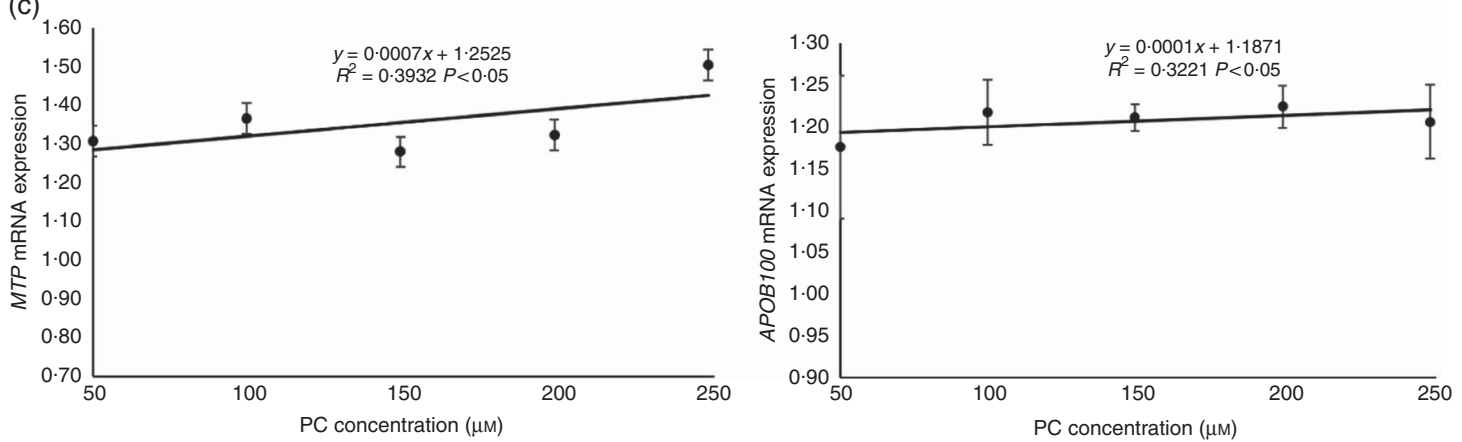

Fig. 3. Regression analyses about expression of genes related to lipid synthesis (a), fatty acid oxidation (b) and VLDL assembly (c) in primary hepatocytes of large yellow croaker in response to graded concentrations of phosphatidylcholine (PC). Values are means $(n 4)$, with their standard errors. The relative mRNA expression of target genes in the control group was selected as the calibrator. SREBP1, sterol-regulatory element binding protein 1; FAS, fatty acid synthase; SCD1, stearoyl-CoA desaturase 1; DGAT2, acyl-CoA: diacylglycerol acyltransferase 2; CPT1, carnitine palmitoyltransferase 1; ACO, acyl-CoA oxidase; MTP, microsomal TAG transfer protein; APOB100, apo B100. 

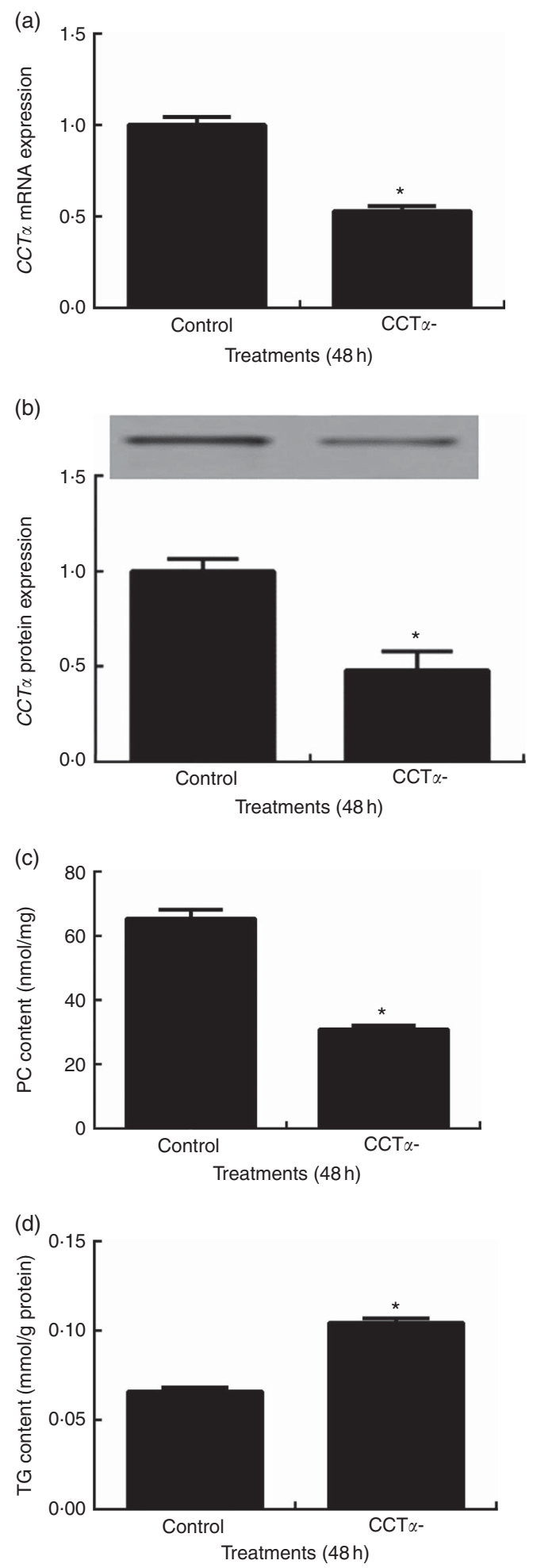

Fig. 4. Effects of CTP: choline phosphate cytidylyltranferase $a$ (CCTa) knockdown on CCTa mRNA expression (a), CCTa protein expression (b), phosphatidylcholine (PC) (c) and TAG (d) contents in primary hepatocytes of large yellow croaker. Values are means $(n 4)$, with their standard errors represented by vertical bars. CCTa, CTP: choline phosphate cytidylyltranferase $a$. Significance was evaluated by two-tailed Student's $t$ test. Significant difference compared with the control group: ${ }^{*} P<0.05$.
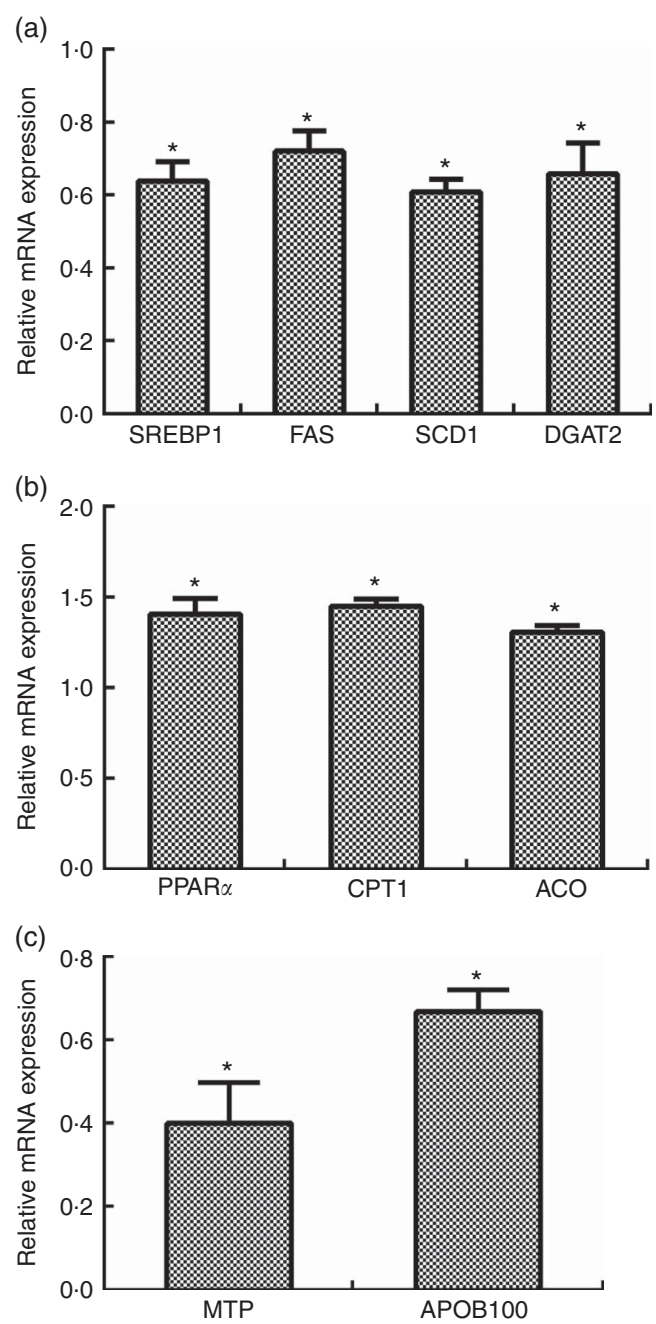

Fig. 5. Effects of CTP: choline phosphate cytidylyltranferase a (CCTa) knockdown on mRNA expression of key genes related with lipid synthesis (a), fatty acid oxidation (b) and VLDL assembly (c) in primary hepatocytes of large yellow croaker. Values are means $(n 4)$, with their standard errors represented by vertical bars. The relative mRNA expression of target genes in the control group was selected as the calibrator. SREBP1, sterol-regulatory element binding protein 1; FAS, fatty acid synthase; SCD1, stearoyl-CoA desaturase 1; DGAT2, acyl-CoA: diacylglycerol acyltransferase 2; CPT1: carnitine palmitoyltransferase 1; $A C O$, acyl-CoA oxidase; MTP, microsomal TAG transfer protein; $A P O B 100$, apo B100. Significance was evaluated by two-tailed Student's $t$ test. Significant difference compared with the control group: * $P<0.05$.

in vitro to verify our hypothesis. In the present study, compared with the LL-LP diet, the HL-LP diet significantly increased the mRNA levels of genes encoding key enzymes related with fatty acid uptake ( $L P L, H L, C D 36, F A T P 1$ and FABP11), which were reversed by dietary inclusion of high PL. The increased expression of these genes was assumed to enhance TAG hydrolysis in lipoproteins and fatty acid uptake ${ }^{(20)}$. Thus, these results indicated that high dietary PL might inhibit excessive fatty acid uptake in large yellow croaker liver caused by HL-LP diet at the transcriptional level, resulting in attenuated hepatic lipid accumulation. These results agreed well with the previous study in mammals demonstrating that PC reversed the increased expression of FATP and FABP in orotic acid-induced fatty liver ${ }^{(11)}$. 
In the present study, high dietary PL significantly attenuated the increased $S R E B P 1$ transcript level induced by high lipid. $S R E B P 1$, as a transcription factor, can activate downstream genes dominating different steps of de novo fatty acid synthesis, such as FAS and $S C D 1^{(38)}$. Together with the inhibition of SREBP1 mRNA abundance by high-dose PL, a concomitant marked reduction in the expression of FAS and SCD1 was observed in this study. Similar results were obtained in the studies of Buang et $a l .{ }^{(6)}$, Kabir \& Ide ${ }^{(8)}$, Liu et al. ${ }^{(10)}$ and Liu et $a l .{ }^{(11)}$. In addition, as DGAT2 catalyses a reaction in which long-chain fatty acyl-CoA is covalently joined to diacylglycerol $^{(39)}$ to generate TAG, the suppression of fatty acid de novo synthesis by high-dose PL might reduce the substrates available for TAG production, partially characterised by decreased expression of DGAT2 caused by high-dose PL. In support of this, Ide $e t a l .{ }^{(40)}$ provided direct evidence that dietary soyabean PL appeared to reduce the availability of fatty acids for TAG synthesis in rat liver through a reduction in the rate of incorporation of $\left[1-{ }^{14} \mathrm{C}\right]$ acetate into fatty acids. Overall, these results indicated that PL might reduce hepatic lipid accumulation through inhibiting lipid synthesis at the transcriptional level. However, to our surprise, conflicting results have been observed for the expression of those genes involved in lipid synthesis in in vitro study. The mRNA expression levels of genes related to lipid synthesis, including SREBP1, FAS, SCD1 and DGAT2, increased with the increasing levels of PC. Being different from in vivo study, primary hepatocytes isolated from large yellow croaker were cultured in artificial medium relatively deficient in nutrients, such as TAG, which serves as highly reduced stores of oxidisable energy. Thus, one possible explanation for the discrepancies between in vitro and in vivo studies was that the up-regulation of lipogenic genes in primary hepatocytes might be driven by a compensation mechanism in response to low hepatic TAG contents. Similarly, the depression of lipogenic gene expression in primary hepatocytes by CCT $\alpha$ knockdown might be partially due to a feedback mechanism involving excessive lipid deposition. As comparable results in published articles on the manipulation of lipogenic genes in primary hepatocytes in response to PC were limited, more relevant works should be performed.

In the present study, no significant differences were detected in the mRNA expression of PPAR $\alpha, C P T 1$ and $A C O$ between the LL-LP and HL-LP groups. PPAR $\alpha$ is an important transcription factor regulating the transcription of CPT1 and ACO, both of which were involved in fatty acid $\beta$-oxidation ${ }^{(41)}$. It was reported that $\operatorname{PPAR} \alpha$ deficiency led to massive hepatic lipid accumulation in response to short-term starvation or a high-fat diet $^{(42,43)}$. Thus, compared with the LL-LP group, lack of an increase in fatty acid oxidation in the HL-LP group might result in subsequent abnormal accumulation of TAG in the liver, which was confirmed by the findings of Wang et al. ${ }^{(37)}$ in the same fish species. The results obtained with the mRNA abundance of genes involved in fatty acid oxidation were contrary to our hypothesis. In this study, the mRNA levels of PPAR $\alpha$ and its target genes CPT1 and ACO were markedly lower in fish fed diets with high levels of PL than those fed diets with low levels of PL regardless of dietary lipid levels. Similar to these results, the mRNA expression levels of genes related to fatty acid oxidation, including PPAR $\alpha, C P T 1$ and $A C O$, decreased with the increasing levels of PC. However, previous research in mammals demonstrated that PL or PC derived from soyabean failed to affect $\beta$-oxidation-related gene expression ${ }^{(6,10)}$. These conflicting results might be attributed to different nutritional conditions and animal species. With respect to the present study, it was hypothesised that depression of PPAR,$C P T 1$ and ACO expression might be secondary to lower lipid contents induced by high-dose PL as a feedback mechanism. Similarly, the up-regulation of fatty acid oxidation related genes caused by CCT $\alpha$ knockdown was probably owing to a feedback mechanism in response to massive lipid accumulation as well.

The export of lipids in liver to periphepatic tissues is mainly achieved by VLDL ${ }^{(44)}$. PL is a key component of VLDL in both mammals and fish ${ }^{(45,46)}$. Furthermore, consistent with most vertebrates, PC appears to be the predominant PL class in fish lipoproteins ${ }^{(47,48)}$. Many studies in mammals have demonstrated that PL or PC play important roles in maintaining normal assembly and secretion of VLDL ${ }^{(12-15)}$. A significant reduction in the appearance of lipoprotein particles in the lamina propria and in the size of such particles was observed in gilthead sea bream fed no soyabean PL-supplemented diets ${ }^{(49)}$. However, no study about the mRNA expression of key genes involved in VLDL assembly in response to PL or PC has been reported yet. Besides lipids, the assembly of VLDL requires APOB100 and $\mathrm{MTP}^{(50)}$. MTP binds and chaperones lipids to the nascent APOB100 to prevent it from aberrant folding and degradation by proteasomes, which is directly related to the number of VLDL ${ }^{(51-53)}$. In the present study, the results demonstrated that the absence of enough PC could inhibit the expression of MTP and $A P O B 100$ at the transcriptional level. Moreover, additional PL or PC might facilitate VLDL assembly by increasing the expression of MTP and $A P O B 100$, which could eventually promote hepatic lipid exportation and subsequently attenuate abnormal hepatic lipid accumulation. The decreased lipid contents might subsequently regulate lipid synthesis and catabolism as a feedback mechanism as mentioned above in the in vitro study.

In summary, both in vivo and in vitro studies demonstrated that high levels of PL or PC could alleviate hepatic lipid accumulation in large yellow croaker. In addition, suppression of PC synthesis could lead to elevated lipid content in primary hepatocytes. High dietary PL might reverse the HL-LP dietinduced abnormal lipid accumulation in the liver through inhibiting fatty acid uptake and lipid synthesis, together with promoting lipid export at the transcriptional level. The in vitro study suggested that PC might affect the expression of lipid export-related genes ( $M T P$ and $A P O B 100$ ) to manipulate hepatic lipid deposition, which agreed well with the findings obtained in the in vivo study.

\section{Acknowledgements}

The authors are grateful to J. L. Du for his assistance in the feeding experiment.

This research was supported by the National Natural Science Foundation of China (31172425 and 31372541), the National 
Science Fund for Distinguished Young Scholars of China (31525024), AoShan Talents Program (2015ASTP), PhD Programs Foundation of the Ministry of Education of China (20120132110007).

Q. A. and K. M. designed the research; Z. C. conducted the research, analysed the data and wrote the paper. All authors have read and approved the final manuscript.

The authors declare that there are no conflicts of interest.

\section{References}

1. Feng S, Cai Z, Zuo R, et al. (2017) Effects of dietary phospholipidss on growth performance and expression of key genes involved in phosphatidylcholine metabolism in larval and juvenile large yellow croaker, Larimichthys crocea. Aquaculture 469, 59-66.

2. Cohn JS, Wat E, Kamili A, et al. (2008) Dietary phospholipids, hepatic lipid metabolism and cardiovascular disease. Curr Opin Lipidol 19, 257-262.

3. Lee YS, Kim WS, Kim KH, et al. (2006) Berberine, a natural plant product, activates amp-activated protein kinase with beneficial metabolic effects in diabetic and insulinresistant states. Diabetes 55, 2256-2264.

4. Polichetti E, Diaconescu N, De La Porte PL, et al. (1996) Cholesterol-lowering effect of soyabean lecithin in normolipidaemic rats by stimulation of biliary lipid secretion. BrJ Nutr 75, 471-481.

5. Polichetti E, Janisson A, De La Porte PL, et al. (2000) Dietary polyenylphosphatidylcholine decreases cholesterolemia in hypercholesterolemic rabbits: role of the hepato-biliary axis. Life Sci 67, 2563-2576.

6. Buang Y, Wang YM, Cha JY, et al. (2005) Dietary phosphatidylcholine alleviates fatty liver induced by orotic acid. Nutrition 21, 867-873.

7. Shirouchi B, Nagao K, Inoue N, et al. (2007) Effect of dietary omega 3 phosphatidylcholine on obesity-related disorders in obese Otsuka Long-Evans Tokushima fatty rats. J Agric Food Chem 55, 7170-7176.

8. Kabir Y \& Ide T (1995) Effect of dietary soybean phospholipids and fats differing in the degree of unsaturation on fatty acid synthesis and oxidation in rat liver. J Nutr Sci Vitaminol 41, 635-645.

9. Leblanc MJ, Brunet S, Bouchard G, et al. (2003) Effects of dietary soybean lecithin on plasma lipid transport and hepatic cholesterol metabolism in rats. J Nutr Biochem 14, 40-48.

10. Liu X, Xue Y, Liu C, et al. (2013) Eicosapentaenoic acidenriched phospholipids ameliorates insulin resistance and lipid metabolism in diet-induced-obese mice. Lipids Health Dis 12, 1-10.

11. Liu Y, Shi D, Liu Y, et al. (2016) Eicosapentaenoic acidcontaining phosphatidylcholine alleviated lipid accumulation in orotic acid-induced non-alcoholic fatty liver. J Funct Foods 23, 294-305.

12. Fast DG \& Vance DE (1995) Nascent VLDL phospholipids composition is altered when phosphatidylcholine biosynthesis is inhibited: evidence for a novel mechanism that regulates VLDL secretion. Biochim Biophys Acta 1258, 159-168.

13. Yao ZM \& Vance DE (1988) The active synthesis of phosphatidylcholine is required for very low density lipoprotein secretion from rat hepatocytes. J Biol Chem 263, 2998-3004.

14. Jacobs RL, Devlin C, Tabas I, et al. (2004) Targeted deletion of hepatic ctp:phosphocholine cytidylyltransferase alpha in mice decreases plasma high density and very low density lipoproteins. J Biol Chem 279, 47402-47410.
15. Jacobs RL, Lingrell S, Zhao Y, et al. (2008) Hepatic ctp:phosphocholine cytidylyltransferase-alpha is a critical predictor of plasma high density lipoprotein and very low density lipoprotein. J Biol Chem 283, 2147-2155.

16. Lu S, Zhao N, Zhao A, et al. (2008) Effect of soybean phospholipids supplementation in formulated microdiets and live food on foregut and liver histological changes of Pelteobagrus fulvidraco larvae. Aquaculture 278, 119-127.

17. Salhi M, Hernández-Cruz CM, Bessonart M, et al. (1999) Effect of different dietary polar lipid levels and different $n-3$ HUFA content in polar lipids on gut and liver histological structure of gilthead seabream (Sparus aurata) Larvae. Aquaculture 179, 253-263.

18. Hemre GI \& Sandnes K (1999) Effect of dietary lipid level on muscle composition in Atlantic salmon Salmo salar. Aquacult Nutr 5, 9-16.

19. Wang T, Yan J, Xu W, et al. (2015) Characterization of cyclooxygenase-2 and its induction pathways in response to high lipid diet-induced inflammation in Larmichthys crocea. Sci Rep 6, 19921.

20. Yan J, Liao K, Wang T, et al. (2015) Dietary lipid levels influence lipid deposition in the liver of large yellow croaker (Larimichthys crocea) by regulating lipoprotein receptors, fatty acid uptake and triacylglycerol synthesis and catabolism at the transcriptional level. PLOS ONE 10, e0129937.

21. Yoon MS, Sun Y, Arauz E, et al. (2011) Phosphatidic acid activates mammalian target of rapamycin complex 1 (mTORc1) kinase by displacing $\mathrm{fk} 506$ binding protein 38 (fkbp38) and exerting an allosteric effect. J Biol Chem 286, 29568-29574.

22. Folch J, Lees M \& Sloane Stanley GH (1957) A simple method for the isolation and purification of total lipides from animal tissue. J Biol Chem 226, 497-509.

23. Bao QL, Deng YJ, Suo X, et al. (2005) Determinating contents of phospholipids in liposomal gel with molybdenum blue method. Chin J Pharm 3, 305-309.

24. Zuo R, Ai Q, Mai K, et al. (2012) Effects of dietary n-3 highly unsaturated fatty acids on growth, nonspecific immunity, expression of some immune related genes and disease resistance of large yellow croaker (Larmichthys crocea) following natural infestation of parasites (Cryptocaryon irritans). Fish Shellfish Immun 32, 249-258.

25. Cai Z, Xie F, Mai K, et al. (2017) Molecular cloning and genetic ontogeny of some key lipolytic enzymes in large yellow croaker larvae (Larimichthys crocea R.). Aquac Res 48, 1183-1193.

26. Livak KJ \& Schmittgen TD (2001) Analysis of relative gene expression data using real-time quantitative PCR and the $2^{-\Delta \Delta \mathrm{Ct}}$ method. Methods 25, 402-408.

27. Andersen CL, Jensen JL \& Ørntoft TF (2004) Normalization of real-time quantitative reverse transcription-PCR data: a modelbased variance estimation approach to identify genes suited for normalization, applied to bladder and colon cancer data sets. Cancer Res 64, 5245-5250.

28. Vandesompele J, Preter KD, Pattyn F, et al. (2002) Accurate normalization of real-time quantitative RT-PCR data by geometric averaging of multiple internal control genes. Genome Biol 3, 1-12.

29. Tocher DR, Bendiksen EÅ, Campbell PJ, et al. (2008) The role of phospholipids in nutrition and metabolism of teleost fish. Aquaculture 280, 21-34.

30. Poston HA (1990) Effect of body size on growth, survival, and chemical composition of Atlantic salmon fed soy lecithin and choline. Progr Fish Cult 52, 226-230.

31. Hung SSO \& Lutes PB (1988) A preliminary study on the nonessentiality of lecithin for hatchery-produced juvenile white 
sturgeon (Acipenser transmontanus). Aquaculture $\mathbf{6 8}$ $353-360$.

32. Geurden I, Coutteau P \& Sorgeloos P (1997) Effect of a dietary phospholipids supplementation on growth and fatty acid composition of European sea bass (Dicentrarchus labrax L.) and turbot (Scophthalmus maximus L.) juveniles from weaning onwards. Fish Physiol Biochem 16, 259-272.

33. Taylor JF, Martinez-Rubio L, Pozo JD, et al. (2015) Influence of dietary phospholipids on early development and performance of Atlantic salmon (Salmo salar). Aquaculture 448, 262-272.

34. Daprà F, Geurden I, Corraze G, et al. (2011) Physiological and molecular responses to dietary phospholipids vary between fry and early juvenile stages of rainbow trout (Oncorbynchus mykiss). Aquaculture 319, 377-384.

35. Zhao J, Ai Q, Mai K, et al. (2013) Effects of dietary phospholipidss on survival, growth, digestive enzymes and stress resistance of large yellow croaker, Larmichthys crocea larvae. Aquaculture 410-411, 122-128.

36. Carmona-Antoñanzas G, Taylor JF, Martinez-Rubio L, et al. (2015) Molecular mechanism of dietary phospholipids requirement of Atlantic salmon, Salmo salar, fry. Biochim Biophys Acta 1851, 1428-1441.

37. Wang X, Li Y, Hou C, et al. (2015) Physiological and molecular changes in large yellow croaker (Pseudosciaena crocea R.) with high-fat diet-induced fatty liver disease. Aquac Res $\mathbf{4 6}$, 272-282.

38. Debose-Boyd RA \& Brown MS (2001) Expression of sterol regulatory element-binding protein 1c (Srebp-1c) mRNA in rat hepatoma cells requires endogenous LXR ligands. Proc Natl Acad Sci 98, 1477-1482.

39. Cases S, Stone SJ, Zhou P, et al. (2001) Cloning of dgat2, a second mammalian diacylglycerol acyltransferase, and related family members. J Biol Chem 276, 38870-38876.

40. Ide T, Murata M \& Sunada Y (1994) Triacylglycerol and fatty acid synthesis in hepatocytes in suspension isolated from rats fed soybean phospholipids. Biosci Biotechnol Biochem 58, 699-702.

41. Goto T, Lee JY, Teraminami A, et al. (2011) Activation of peroxisome proliferator-activated receptor-alpha stimulates both differentiation and fatty acid oxidation in adipocytes. J Lipid Res 52, 873-884.
42. Kersten S, Seydoux J, Peters JM, et al. (1999) Peroxisome proliferator-activated receptor alpha mediates the adaptive response to fasting. J Clin Invest 103, 1489-1498.

43. Leone TC, Weinheimer CJ \& Kelly DP (1999) A critical role for the peroxisome proliferator-activated receptor alpha (ppar alpha) in the cellular fasting response: the pparalpha-null mouse as a model of fatty acid oxidation disorders. Proc Natl Acad Sci U S A 96, 7473-7478.

44. Sundaram M \& Yao Z (2010) Recent progress in understanding protein and lipid factors affecting hepatic VLDL assembly and secretion. Nutr Metab 7, 1-17.

45. Santulli A, Messina C, Modica A, et al. (1996) Lipid and apolipoprotein composition of lipoproteins of European sea bass (Dicentrarchus labrax L.). Comp Biochem Physiol B $\mathbf{1 1 4}$ 321-326.

46. Sheridan MA (1988) Lipid dynamics in fish: aspects of absorption, transportation, deposition and mobilization. Comp Biochem Physiol B 90, 679-690.

47. Nelson GJ \& Shore VG (1974) Characterization of the serum high density lipoprotein and apolipoproteins of pink salmon. J Biol Chem 249, 536-542.

48. Chapman MJ (1980) Animal lipoproteins: chemistry, structure, and comparative aspects. J Lipid Res 21, 789-853.

49. Liu J, Caballero MJ, Izquierdo M, et al. (2002) Necessity of dietary lecithin and eicosapentaenoic acid for growth, survival, stress resistance and lipoprotein formation in gilthead sea bream Sparus aurata. Fisheries Sci $\mathbf{6 8}$, $1165-1172$

50. Hussain MM, Nijstad N \& Franceschini L (2011) Regulation of microsomal triglyceride transfer protein. Clin Lipidol $\mathbf{6}$, 293-303.

51. Olofsson SO, Boström P, Andersson L, et al. (2009) Lipid droplets as dynamic organelles connecting storage and efflux of lipids. Biochim Biophys Acta 1791, 448-458.

52. Gordon DA \& Jamil H (2000) Progress towards understanding the role of microsomal triglyceride transfer protein in apolipoprotein-b lipoprotein assembly. Biochim Biophys Acta 1486, 72-83.

53. Vergès B (2010) Abnormal hepatic apolipoprotein b metabolism in type 2 diabetes. Atherosclerosis 211, 353-360. 